\title{
Experiences, Choice and Well-Being: An Economics of Psychological Energy
}

\author{
David Allen Axelrod, Ph.D. \\ Montclair State University, Montclair, NJ,United States, axelrodd@montclair.edu
}

\begin{abstract}
This paper proposes an economic model of psychological energy used toward the production of experiences. A review of ideas at the nexus of economics and psychology, and how they lead to the thesis of this paper, is presented. A simple mathematical economic model is developed, with two main uses of psychological energy toward well-being. These are the generation of impressions (inward experiences that are sense-like) and expression (outward experiences that are action-like). Choosing is understood as investing energy to change the probabilities of an outcome. The model optimizes energy use between intensity of impressions and capacity for expression. For a fixed energy level, as experiential intensity increases resources are substituted out of decision making and implementation, leading to choices of lower utility. If the material losses are substantial during an experience, the share of psychological energy used to modify impressions will increase, and away from influencing seemingly random external outcomes. Over multiple periods, this generates a feedback loop where the person feels increasingly disempowered, and thus less concerned about making better choices. This feedback loop can be stopped by an external entity providing sufficient resources for the person to experience greater expression.
\end{abstract}

KEYWORDS: Impression, Expression, Internal Environment, Poverty Trap

\section{Introduction}

The premise of this paper is that the essence of economic activity is the generation of experiences. Our choices influence these experiences in two important ways. The first is over the expressions to manifest through our behavior. The second is over how to interpret events that leave the impressions we savor, and which become our memories and foundations for future experiences. It is in this balance of impressions and expressions, given our finite material and psychological resources, that our well-being is generated. The standard conception of economic activity is that of production, exchange and consumption, with a particular focus on material goods and services. However, experiences are more than this; they can be creative, spiritual, social, and transformative, among other qualities. In the same way that Lancaster [1966] conceived of decision making as choice over attributes of goods, we can consider it as choice over aspects of experiences.

Pine and Gilmore's The Experience Economy (2011) delves deep into a world of staging events of value for an audience. They explicate a model describing the progression of economic value from commodities, through goods and services, into experiences. For them "Work is Theater" and the retail shop is replaced by a performance stage. They describe experiences along two axes: passive-active and absorption-immersion. An experience is active if the consumer influences the performance, and passive otherwise. Absorption describes how completely an experience occupies a person's attention. Immersion describes how fully a person becomes part of the experience. This leads them to write of transformations as the further culmination of economic value, where the customer is the product.

If Pine and Gilmore highlight the economic value of experiences, Mulainathan and Shafir (2013) remind us of the costs involved. They consider the behavioral economics and cognitive psychology involved with scarcity, as we face situations of finite time and resources. They start by elaborating on the distinction between "tunneling" and "focusing." Tunneling occurs when we ignore potential feasible alternatives, once we have committed to an action. Focusing is our ability to invest our mind toward an outcome, while remaining open to potential contingencies and emergent alternatives. They describe a finite bandwidth available for "cognitive capacity" and "executive control" that drives our 
behavior and worldview. However, this scarcity of mind is distinguished from a scarcity mindset. While all of us must contend with the first, it's the latter that some will struggle against. The authors present poverty as both external and internal (whether individual or organizational), due to what they refer to as a "bandwidth tax", the cost in mental space. The limited internal resources leads to myopic choices.

Laajaj (2012) presents the results of a study where impoverished farmers are provided financial resources. His hypothesis, which the results support, is that the amount of resources available affects not only what can be bought, but also how decisions are made. In particular, as the farmers were provided greater aid, they made decisions with broader time horizons. Those in poverty are limited in planning for the future, which magnifies that future's uncertainty. Axelrod (1990) demonstrates how scarcity on the ability to process uncertainty would limit how far the future is considered in a present value calculation, beyond which the consequences would be ignored.

To deepen the connections between economics, experiences, choice and well-being, it will be insightful to consider Tibor Scitovsky's The Joyless Economy. He writes, "Culture is the preliminary information we must have to enjoy the processing of further information" (Scitovsky 1976, 226). This sentence captures two essential elements. First, it describes how we live in an information economy. The utility, and value, of a set of information is contingent on how we can process and understand it. The blossoming of the information economy, with its culture of data exchange and accumulation, and the various software and hardware required to partake in it, has been right on cue. Second, culture can also be understood as the psycho-sociological space within which economic matter moves, is measured and, ultimately, is experienced. To a hammer, everything seems like a nail; to an artist a nail can be anything.

The 19th century economist Leon Walras succinctly expresses this distinction. Walras (1954) described persons as "self-conscious" and "self-determining." Things are "not self-conscious" and are at the command of persons. This dichotomy presumes that persons and things are clearly defined, and that personhood is inviolable. This presumption is useful for the sake of developing a novel theory; however, it bypasses one of the essential reasons for having economic activity - to experience ourselves as persons through self-expression. In other words, an economy produces persons, as well as things. Walras goes on to describe the distinction between intensive and extensive utility. The marginalist revolution, of which he was one of its founders, incorporated the subjective as part of its explanation of economic behavior. It is the psychological space which modern economics ultimately relies on to explain consumer behavior.

The relationship between space and matter can also be understood in the nature of savoring. Scitovsky $(1976,184)$ raises the question, "Is it Too Dull?", and writes, "Few people will admit to themselves, or even be conscious of, their lack of skill in savoring food." Part of his argument refers to the blandness of American food relative to European food. One can immediately appreciate the role of space in welfare and enjoyment by considering a simple experiment. Take a dish of your favorite food. From this, have a small bite, and focus on its flavor. Next, take a slightly larger bite, and again allow yourself to focus on its flavor. Repeat this until you have had a big bite, so large that it almost fills your mouth. Does it still have the same flavor? Do you get the same enjoyment of its taste? Is your most flavorful bite size smaller than the largest bite you could take? It is not just matter, but also the space to savor it, that affects our experiences. One can easily imagine a utility function with volume of space/time, and mass of matter, as variables. Indeed, households with income constraints face a tradeoff between how big a house to live in, and how much they can fill it with. Becker (1965) explicitly introduces time as part of consumption. The implication is direct. When an individual (or society) stuffs themselves so that they have no space to savor what they have consumed, well-being can decrease. This does not negate the typical economic assumption that more is preferred to less. Rather, it is the recognition that there is a trade-off between having more things, and having the space to enjoy them. It is the need for this "inner" space, for room to reflect upon sensation and to appreciate its 
interconnectedness with other experiences, which limits the applicability of standard economics. It is the space between bits of information that enables us to have information at all.

Lancaster $(1966,132)$ writes, "All intrinsic properties of particular goods, those properties that make a diamond quite obviously something different from a loaf of bread, have been omitted from the theory, so that a consumer who consumes diamonds alone is as rational as a consumer who consumes bread alone, but one who sometimes consumes bread, sometimes diamonds (ceteris paribus, of course), is irrational." His recognition of the importance of the essential properties of an external good underscores the distinction between what is being produced and sold, and what consumers are seeking, the ability to satisfy desires, needs and wants. It is not surprising to see the contemporaneous writings of Lancaster and Scitovsky attempt to fill the void of economic theory at the time. One approach focused on the intrinsic attributes of the good, the other the intrinsic attributes of the consumer. In essence, the simple awareness of the intrinsic attributes of a good must be developed before they can be appreciated. This is about the relationship between the good and the consumer. With respect to the experience of well-being, it is as if the consumed and the consumer are complementary economic goods. From a neuroeconomic perspective, it is the availability of both neurotransmitters (like dopamine) and binding receptors that is required for an experience to occur (Doidge 2012).

If we understand that the ultimate outputs of an economy are experiences, then we can speak of the attributes of these experiences. That experience can be of eating tasty food, watching a compelling movie, scoring a goal in a soccer match, playing music on a piano, or taking a relaxing walk along a canal (among many others). Deeper, though, is the distinction between consuming and choosing. Part of our experiences are the attributes of who we are. When I play electric bass, one part of the experience is the sound that is produced. However, another part of the experience is the feeling of performing, of creative expression, that I have choice over what I am doing, and that it is reflected in how the material world manifests. Even if I do not play a composition as well as others might, it can be more satisfying to play it myself, than to sit and watch someone else play. In both cases I can feel a sense of well-being, yet one is not quite as fulfilling as another. Csikszentmihalyi (1990) might refer to the state of mind associated with this expressive satisfaction as "Flow." The sounds I make when playing can leave impressions within those who listen (including myself). The energetic flow of having chosen to play those sounds is one of expression. We seek to optimize the balance of these two.

This distinction is also similar to the one that Herbert Simon (1976) makes between substantive and procedural rationality. The former identifies rationality based on what one chooses (e.g. what one consumes). The latter identifies rationality based on how one chooses. The first is about the choice made being rational, the second about the chooser being rational. In his Models of Man (Simon 1957), bounded rational agents are limited in processing information. This relates back to Scitovsky's quote above. Culture is, in effect, one of the bounding factors, and is essential in determining what alternatives are ignored and how they are valued. Some of the attributes of culture are the resources available, such as material wealth. The more one spends on making decisions the less is available to implement them. However, if one uses too little resources in making decisions, the remaining resources may be used so inefficiently as to lead to an inferior outcome. Again, one can imagine another utility function where one input is the space to make decisions, and the other is the material with which to implement them. Castellani, Di Giovinazzo and Novarese (2010) discuss this relationship between procedural rationality and happiness.

The value of expression in relation to economic activity has its recognition over the spectrum of economic philosophy. Fromm $(1961,34)$ summarizes Karl Marx's description of labor as "not only a means to an end - the product - but an end in itself, the meaningful expression of human energy." Von Mises $(1996,22)$ writes that causality is a prerequisite of acting, and, "Only a man who sees the world in the light of causality is fitted to act." Whether Protestant work ethic (Weber 1930), or the artist's "queer divine dissatisfaction" (Graham in De Mille 1991), it is the embrace of oneself as a force of 
nature that unleashes both the creation and production of goods, and their valorization, essential for an economic world-view. The moment of choice is valuable not only because of its consequences (the range of options in the future), but also as an end in itself. A day of rest at the end of the workweek gives us space to savor life. It is that much sweeter when we have worked diligently, such that what surrounds us is easily imagined as our own handiwork, and what we find within delights us as well.

Scitovsky $(1976,262)$ also writes, "People's desire for the uniqueness of a painting, an art object, a dress, or any other possession therefore, however snobbish it may seem, is soundly based on a desire for maximum novelty and stimulus enjoyment." If commodities and consumers are complementary, then we might expect the uniqueness of self (and its perpetual reinvention) to also provide novelty and stimulus. If persons are self-determining, then in the absence of innovation in the environment, especially the socio-economic environment, we might expect this drive for novelty to find internal expression as a change in self and perception. Thus, how a person filters external stimulus and generates impressions is not just dynamic, it is also an outcome of decision-making and choice, even if arrived at unconsciously. In a world of bounded rationality and adaptable minds, it becomes rational to adapt preferences and perception to fit a world that seemingly has become static. If the food stays the same, novelty and stimulus can be found in practicing how to taste differently. In the most extreme case, one might use hallucination and fabrication to create the desired novelty (McKenna 1993). The minimalism of Zen is the maximization of the space with which to savor life.

However, if there can be commodity fetishism, there can also be consumer fetishism. One could argue that the Von Mises' conception of consumer sovereignty is exactly that. Scitovsky $(1962,248)$ writes, "The economist could wash his hands of value judgments only if the public's preferences were really given and he could accept them as such." Thus, the ability to produce consumers (or at least their preferences) is as important to economic growth as the ability to produce goods. Indeed, the essence of marketing, what makes it useful and valuable, is just that. In a world of scarce resources, the full cost of a sustainable culture must include the energy used both for producing the tokens of culture (art work, cuisine, sports gear, musical instruments, weapons, etc.), and the capacity to use and appreciate them. It takes both objective expression (extensive utility) and subjective impression (intensive utility) for the manifestation of a culture and its associated sense of well-being.

All of this leads to an economics of psychological energy. Since, as Doidge (2012) writes, the brain has neuroplasticity and can change itself, then the mind literally faces a choice between using energy to trigger muscular responses that can change its external environment and/or to trigger glialneural responses that change its internal environment. One suggested physical mechanism is glucose available in the brain (Gailliot et al. 2007). Well-being becomes a holonomic experience that is dependent on the interplay of expression and impression. Holonomics (Robinson and Robinson 2014) elaborates on this in terms of business and ecology. Sustainable well-being is dependent on the economic resources to perpetuate an equilibrium of mind, even as the world appears chaotic.

\section{Experiences, Impressions and Expressions}

Experiences are events that leave impressions and/or give expression. An impression is relative to an observer, and can be understood as energy impressed upon that observer. It is sense-like. An expression is relative to an actor, and can be understood as energy expressed from them. It is action-like. Wellbeing is a state that can arise from these impressions and expressions. For a musician, what they play/sing are expressions, what is heard and felt are impressions. To those familiar with yogic philosophy (Taimini, 1961), the distinction between impressions and expressions is similar to that of jnanendriyas (cognitive senses) and karmendriyas (active senses) as described by Mishra (1997). Given that the practice of yoga involves mental focus, it can be understood as an applied economics of the mind involving choice over how to use the resources of awareness and consciousness. A self-conscious decision maker is one for whom their expressions and impressions are entangled. 
The capacity to express oneself is, in essence, a feasible alternative set. Thus, preferences describe a hierarchy of expression. This is similar to the hierarchy of needs described by Maslow (1943). The capacity for, and preferences over, impression, can be understood similarly. Well-being is a realization of these preferences and capacity. Further, such capacities are dependent on the history of choices made. For example, the capacity to express oneself as a musician depends on the choice to practice and study an instrument, which in turn is dependent on the choices made by others to have produced instruments, to have taught music, to have provided you with a place to practice, and to have endowed you with enough raw talent to make it all worthwhile. The same holds for impressions.

It is important to recognize that the manifestation of the material world is an outcome of many entities expressing themselves simultaneously. When a person makes a choice, they are only influencing that outcome. The more powerful a person, the closer the world expresses their choice. Under certain circumstances, a person chooses as if they are the sole influence. For example, if you are home alone for the evening, and well-stocked with ice cream, your choice to eat ice cream will be almost certainly realized. Thus, choice is an expression that changes the probabilities of events. We can make a conjecture: the greater the energy of expression, the more likely its realization.

Since choice is an expression of preferences, all else equal, the greater the energy used to make a choice, the more preferred the expected outcome. Consider how one will spend more time and effort buying a car (researching brands and models, dealers, reviews, etc.) expecting that a better choice will be made. One may still end up buying a car while using little effort to make a choice; however, it is unlikely to be the best deal one could imagine having made. Further, if one makes no effort to choose buying a car, one could still end up owning a car, but only by chance (e.g., gift or bequeathal). What is important, from an economic perspective, is that we can understand that the expected utility of a choice is a function not only of the goods and services being considered, but also the resources used to make that choice.

One property of experience can be self-consciousness. In this case the feasible alternative set is not of material economic goods and services, but of how to perceive one's self as a decision maker. As such this choice influences economic activity (in a material sense) and well-being (as a physiological and psychological state). This has implications for how a decision maker frames and views their choices. For example, consider when people say, "Choose from your heart and not your head." The first is an appeal to the emotional and subjective. It is the strength and intensity of feelings that energizes a chosen alternative. The latter is an appeal to the rational and objective. It is the facts and their logical implications that decide upon an alternative. Choosing "from the heart" can be understood as substantively rational if emotional states are understood as another type of good. In other words, the well-being (W) of a person is a function of psychological and economic states (other factors can be included, such as sociological status and connections). One way to imagine this is the psychological state as the interface between the material world and the experience of well-being. The implication is that, under particular conditions, choosing emotionally with less pecuniary benefit is preferable to choosing rationally with more pecuniary benefit. Whether there is regret later on depends on the futureexperienced cost of having chosen emotionally.

\section{Model}

In the model to be presented, psychological energy can be used both to transform the internal space from which impressions arise, and to focus on which expressions are chosen. In the context of bounded rationality, there is a trade-off between resources used to experience and resources used to choose. A useful analogy is the distinction between choosing a radio frequency to listen to, and how much to amplify that signal. The latter influences how intensely the signal is experienced. As experiential intensity increases resources are substituted out of rational processing, leading to choices that tend to 
have lower utility (less preferred). However, the over-all sense of well-being can be enhanced if the increase in intensity more than compensates for the reduced utility.

Before presenting the mathematical model, the essential relationships underlying them are displayed in Figure 1. The decision maker is faced with an external environment (an amalgam of material and social factors), an internal environment (which includes, but is not limited to, beliefs, paradigms, filters, instincts, habits, reasons and available energy), and an interface between the two (typically understood as the body, and especially the brain). The state of the external environment triggers events toward the person (inputs), which go through processing, and generate impressions and energy. Impressions go through another round of processing, and generate expressions (dependent on energy available). These expressions become events from the person (output), which then impact the interface and external environment.

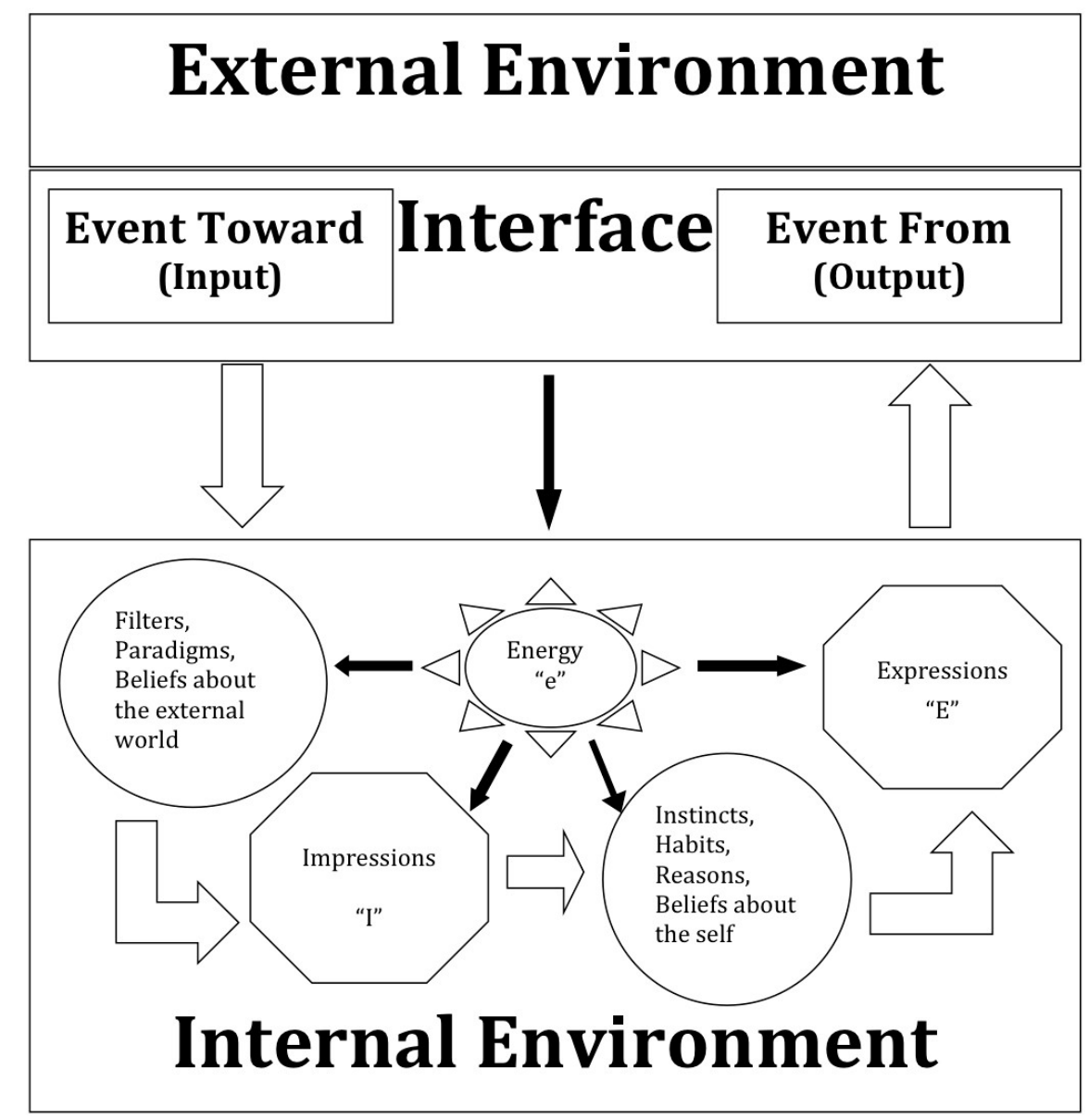

Figure 1: Representation of Environments, Energy, Impressions and Expressions

In our mathematical model we make the following assumptions:

A) Psychological resources are finite. Specifically, the psychological energy available to transform the internal environment (savoring an impression), and/or transform the external environment (choose an expression) is limited. This is represented by the following constraints:

$\mathrm{e}_{\mathrm{I}}+\mathrm{e}_{\mathrm{E}}<=\mathrm{e}, \quad \mathrm{e}_{\mathrm{I}} \geq 0, \quad \mathrm{e}_{\mathrm{E}} \geq 0$ 
where $e$ is the total energy available, $e_{\mathrm{I}}$ is the energy used for receiving impressions and $\mathrm{e}_{\mathrm{E}}$ is the energy used for giving expressions. One could also conceive these as energy internalized and energy externalized. Although not developed in this paper, a multi-person model might include energy exchange and dynamic internal/external boundaries, potentially useful for discussing organizational and social psychology.

B) The intensity of experiencing an event increases, at a decreasing rate, as more resources are used to amplify this intensity. We can also conceive this as the volume of internal space inflating at a decreasing rate, as energy internalized increases. A simple relationship would be:

$\mathrm{I}\left(\mathrm{e}_{\mathrm{I}}\right)=\left(\mathrm{e}_{\mathrm{I}} / \alpha\right)^{\beta}$, where $\alpha>0,0<\beta<1 \quad(\alpha$ is a normalization constant $)$

C.1) The expected utility derived from an outcome (x) depends on the resources used to choose the outcome, i.e., the more resources that are available to choose an outcome, as an expression of one's preferences, the greater the utility of the outcome (a form of bounded rationality).

C.2) If no resources are used to make a decision, the expected utility of a random (possibly habituated) event is $\mathrm{U}_{0}$. A simple formula consistent with C.1 and C.2 would be:

$\mathrm{U}\left(\mathrm{x}, \mathrm{e}_{\mathrm{E}}\right)=\left(\mathrm{U}_{0}(\mathrm{x})+\phi^{*} \mathrm{e}_{\mathrm{E}}\right), \quad$ where $0<\phi$

We use this formula to allow for the possibility that utility could be negative, if $U_{0}$ is negative and insufficient energy is used to improve the situation. Let ${ }_{\mathrm{E}}{ }^{\mathrm{m}}$ be the minimum level of expressive energy required to increase expected utility to 0 . Thus,

$\mathrm{e}_{\mathrm{E}}^{\mathrm{m}}=-\mathrm{U}_{0} / \phi$ when $\mathrm{U}_{0}<0$, and 0 otherwise

D) The Well-being of an individual is a function of the Intensity of experiencing an event and the Utility of manifesting an event. A simple multiplicative form would be:

$\mathrm{W}=\mathrm{I} * \mathrm{U}=\left[\left(\mathrm{e}_{\mathrm{I}} / \alpha\right)^{\beta}\right] *\left(\mathrm{U}_{0}+\phi^{*} \mathrm{e}_{\mathrm{E}}\right)$

The above assumptions can be incorporated in the Lagrangian function:

$\mathrm{L}=\left[\left(\mathrm{e}_{\mathrm{I}} / \alpha\right)^{\beta}\right] *\left(\mathrm{U}_{0}+\phi^{*} \mathrm{e}_{\mathrm{E}}\right)+\lambda *\left(\mathrm{e}-\mathrm{e}_{\mathrm{I}}-\mathrm{e}_{\mathrm{E}}\right)$

If we take the first-order necessary condition to maximize $\mathrm{L}$ we get:

$$
\begin{aligned}
& \mathrm{dL} / \mathrm{de}_{\mathrm{I}}=(\beta / \alpha)\left(\mathrm{e}_{\mathrm{I}} / \alpha\right)^{\beta-1}\left(\mathrm{U}_{0}+\phi^{*} \mathrm{e}_{\mathrm{E}}\right)-\lambda=0 \\
& \mathrm{dL} / \mathrm{de}_{\mathrm{E}}=\phi\left(\mathrm{e}_{\mathrm{I}} / \alpha\right)^{\beta}-\lambda=0 \\
& \mathrm{dL} / \mathrm{d} \lambda=\mathrm{e}-\mathrm{e}_{\mathrm{I}}-\mathrm{e}_{\mathrm{E}} \geq 0
\end{aligned}
$$

From this we derive,

$(\beta / \alpha)\left(\mathrm{e}_{\mathrm{I}} / \alpha\right)^{\beta-1}\left(\mathrm{U}_{0}+\phi^{*} \mathrm{e}_{\mathrm{E}}\right)=\phi\left(\mathrm{e}_{\mathrm{I}} / \alpha\right)^{\beta}$

and finally, when e $>-\mathrm{U}_{0} / \phi=\mathrm{e}_{\mathrm{E}}^{\mathrm{m}}$ and $\mathrm{e}>\left(\beta \mathrm{U}_{0} / \phi\right)$ : 
$\mathrm{e}_{\mathrm{I}}=(\beta /(1+\beta)) *\left(\mathrm{e}+\left(\mathrm{U}_{0} / \phi\right)\right)$

$\mathrm{e}_{\mathrm{E}}=(1 /(1+\beta)) *\left(\mathrm{e}-\left(\beta \mathrm{U}_{0} / \phi\right)\right)$

However, corner solutions can exist. If $\mathrm{e}>\mathrm{e}_{\mathrm{E}}{ }^{\mathrm{m}}$ and $\mathrm{e} \leq\left(\beta \mathrm{U}_{0} / \phi\right)$, then $\mathrm{e}_{\mathrm{E}}=0$ and $\mathrm{e}_{\mathrm{I}}=\mathrm{e}$. If $\mathrm{e} \leq \mathrm{e}_{\mathrm{E}}{ }^{\mathrm{m}}$, then $\mathrm{e}_{\mathrm{I}}=0$ and $\mathrm{e}_{\mathrm{E}}=\mathrm{e}$ (although $\mathrm{W}=0$ for any $\mathrm{e}_{\mathrm{E}}$ ). An interpretation of $\mathrm{e}_{\mathrm{I}}=0$ is the person collapsing to a "perfectly" objective entity - something without internal space to be subjective.

What does this mean? If the person is endowed with enough psychological energy they will diversify it between expanding the space to savor impressions and flowing the resources to materialize expression. If that energy is small, and they face a positive random outcome, they will invest all of it toward savoring the experience, and none of it toward choosing a better expression. If a person has little energy and faces a negative random outcome, they will avoid experiencing the world, and are unlikely to do anything to improve it.

\section{Dynamic Model with Endogenous Energy}

Let us now consider the implications of the model, in a multi-period world. What happens to total energy (e), and its distribution, over time? To consider this we must hypothesize how e changes. One place to start is by assuming that the level of utility in the present moment affects how much energy is available in the next. This seems reasonable, since part of the meaning of economic utility is producing the energy to express oneself. So, we add the following assumptions:

E.1) There is a level of utility, $U^{*}$, in the present moment which generates the same psychological energy for the next moment as the present.

E.2) There a maximum level of psychological energy, $\mathrm{e}^{*}$, that can be held.

We use the following formula:

$e_{t+1}=\operatorname{Max}\left[e^{*},\left(U_{t} / U^{*}\right)^{v} * e_{t}\right], \quad 0<v<1$, when $U_{t}>0$ (otherwise $e_{t+1}=0$ )

Where $U_{t}$ is the level of utility in period t. Substituting in for $U_{t}$ we then get

$\mathrm{e}_{\mathrm{t}+1}=\operatorname{Max}\left[\mathrm{e}^{*},\left(\left(\mathrm{U}_{0}+\phi^{*} \mathrm{e}_{\mathrm{E}, \mathrm{t}}\right) / \mathrm{U}^{*}\right)^{v} * \mathrm{e}_{\mathrm{t}}\right]$

The situation $\mathrm{e}_{\mathrm{t}+1}=0$ means no psychological energy is available. Various scenarios are summarized in Table 1. Note that if $\mathrm{e}_{\mathrm{t}}>\left(\beta \mathrm{U}_{0} / \phi\right)$ and $\mathrm{U}_{0}>0$, then $\left(\left(\mathrm{U}_{0}+\phi^{*} \mathrm{e}_{\mathrm{t}}\right) /(1+\beta)\right)>\mathrm{U}_{0}$.

What does this mean? First, if $\mathrm{U}_{0}$ is negative and the starting energy is sufficiently large to enable positive utility, the generation of energy in the next period is similar to the case for non-negative $\mathrm{U}_{0}$, except that the required energy is relatively higher. In other words, the bar is set higher, but if crossed the results are similar. Second, if the energy is not enough to generate positive utility in any circumstance, the result is that there is no energy for the next period, and the person will become psychologically "dead", an object without any inner space or resources. Third, for a finite mind there is an upper limit for energy increase. In this model, without intervention, psychological energy would continue at $\mathrm{e}^{*}$. A future model will consider other possibilities. An example will be an energy cost for discharging energy beyond the maximum. This could be interpreted as a repair, or healing, cost for overload. Thus, sustainable well-being would depend on approaching, but not reaching $\mathrm{e}^{*}$; otherwise, going beyond the maximum could leave a person with less energy in the future. This could describe individuals with some forms of epilepsy, or bipolar functionality. Another possibility is the surplus energy beyond e* overflows into other persons. This could describe those who are inspirational. 
Table 1: Change in Psychological Energy over Time

\begin{tabular}{|c|c|c|c|c|}
\hline \multicolumn{2}{|c|}{$\mathrm{U}_{0} \geq 0$} & \multicolumn{2}{|c|}{$\mathrm{U}_{0}<0$} & \\
\hline $\mathrm{e}_{\mathrm{t}} \leq\left(\beta \mathrm{U}_{0} / \phi\right)$ & $\mathrm{e}_{\mathrm{t}}>\left(\beta \mathrm{U}_{0} / \phi\right)$ & $\mathrm{e}_{\mathrm{t}} \leq\left(-\mathrm{U}_{0} / \phi\right)$ & $\mathrm{e}_{\mathrm{t}}>\left(-\mathrm{U}_{0} / \phi\right)$ & Energy $t$ \\
\hline $\mathrm{U}_{0}<\mathrm{U}^{*}$ & $\left(\mathrm{U}_{0}+\phi^{*} \mathrm{e}_{\mathrm{t}}\right) /(1+\beta)<\mathrm{U}^{*}$ & All cases & $\left(\mathrm{U}_{0}+\phi^{*} \mathrm{e}_{\mathrm{t}}\right) /(1+\beta)<\mathrm{U}^{*}$ & Decrease \\
\hline $\mathrm{U}_{0}=\mathrm{U}^{*}$ & $\left(\mathrm{U}_{0}+\phi^{*} \mathrm{e}_{\mathrm{t}}\right) /(1+\beta)=\mathrm{U}^{*}$ & N.A. & $\left(\mathrm{U}_{0}+\phi^{*} \mathrm{e}_{\mathrm{t}}\right) /(1+\beta)=\mathrm{U}^{*}$ & Same \\
\hline $\mathrm{U}_{0}>\mathrm{U}^{*}$ & $\left(\mathrm{U}_{0}+\phi^{*} \mathrm{e}_{\mathrm{t}}\right) /(1+\beta)>\mathrm{U}^{*}$ & N.A. & $\left(\mathrm{U}_{0}+\phi^{*} \mathrm{e}_{\mathrm{t}}\right) /(1+\beta)>\mathrm{U}^{*}$ & Increase \\
\hline
\end{tabular}

† Decrease bounded by 0 , Increase bounded by e*

\section{Conclusion}

This paper attempts to show how conceiving economic activity as generating experiences can be used to create a simple model of well-being, and the distribution of psychological energy toward experiencing and choosing, using the concepts of impressions and expressions. The results suggest that the lower the energy available, the more likely an individual will use it to savor the impressions from a seemingly randomly supplied material world. However, the greater the energy and/or economic endowment, the more an individual will use that energy to express themselves in the world. A further implication is that with greater resources comes the tendency to make choices that can reinforce expression that leads to a growth in psychological energy, a virtuous cycle. On the other hand, those who start with little psychological energy, and face a weak (or bleak) economic condition, will tend to avoid expressing themselves, leading to less energy and well-being, until they shut down at the end of a vicious cycle. These cycles can be broken by a change of fortune, including the behavior of others.

One could enhance the realism, and applicability, of these models by including other elements (e.g., intertemporal choice, social interaction and further feedback structures between economic resources and psychological energy). Of particular interest would be how $\mathrm{U}_{0}$ and $\mathrm{U}^{*}$ change with wealth and well-being (a topic for another paper). Although the model is presented as the inner workings of an individual, the same principles and insights could be ascribed to social groups and their drifts toward empowerment or myopia.

\section{Acknowledgments}

This paper is a based on a presentation at the CREAM conference in honor of Tibor Scitovsky, June 2012, Gaeta, Italy. I would like to thank Sergio Nistico, Marina Bianchi, Carol Boyer, and Scott Axelrod, among many others, who have provided helpful comments and insights. I would also like to thank Jim Leitner for support and encouragement.

\section{References}

Axelrod, D. 1990. "The Rationality of Positive Time Preference." Three Essays on Latency in Economics and Decision Making, Doctoral Dissertation, Rutgers University.

Becker, G. S. 1965. “A Theory of the Allocation of Time.” Economic Journal 75(299): 493-517.

Csikszentmihalyi, M. 1990. Flow: The Psychology of Optimal Experience. NY, NY: Harper \& Row.

Castellani, M., Di Giovinazzo, V., and Novarese, M. 2010. "Procedural Rationality and Happiness." Journal of SocioEconomics 39 (3): 376-383.

De Mille, A. 1991. Martha: The Life and Work of Martha Graham. NY, NY: Random House.

Fromm, E. 1961. Marx's Concept of Man. Indiana University: F. Ungar, Pub. Co.

Gailliot, M.T. et al. 2007. "Self-Control Relies on Glucose as a Limited Energy Source." Journal of Personality and Social Psychology 92: 325-336.

Laajaj, R. 2012. "Closing the eyes on a gloomy future: Psychological causes and economic consequences." Pacific Development Economics Conference, University of California, Davis.

Lancaster, K. J. 1966. “A New Approach to Consumer Theory.” Journal of Political Economy 74: $132-157$.

Marx, K. 1977. Capital: Volume One. Translated by Ben Fowkes, NY, NY: Vintage Books.

McKenna, Terence. 1993. Food of the Gods: the Search for the original Tree of Knowledge. NY, NY: Bantam. 
Mishra, R. 1997. The Textbook of Yoga Psychology. Baba Bhagavandas Publication Trust: Monroe, NY.

Mulainathan, S. and Shafir, E. 2013. Scarcity, NY, NY: Picador.

Pine, B. and Gilmore, J. 2011. The Experience Economy, Boston, MA: Harvard Business School Publishing.

Robinson, S. and Robinson, M. 2014. Holonomics. Edinburgh, UK: Floris Books.

Scitovsky, T. 1962. "On the Principle of Consumers' Sovereignty." The American Economic Review, 52(2), Papers and Proceedings of the Seventy-Fourth Annual Meeting of the American Economic Association (May, 1962), $262-268$.

Scitovsky, T. 1976. The Joyless Economy: An Inquiry into Human Satisfaction and Consumer Dissatisfaction. USA: Oxford University Press.

Simon, H.A. 1957. Models of Man: Social and Rational. NY, NY: John Wiley and Sons, Inc.

Simon, H.A. 1976. "From Substantive to Procedural Rationality." 25 Years of Economic Theory, pp. 65-86, US: Springer.

Taimini, I.K. 1961. The Science of Yoga. Wheaton, IL: The Theosophical Publishing House.

Von Mises, L. 1996. Human Action. (4 ${ }^{\text {th }}$ Ed.). San Francisco, CA: Fox \& Wilkes.

Walras, L. 1954. Elements of Pure Economics. English translation by W. Jaffé. Homewood, IL: Richard Irwin.

Weber, M. 1930. The Protestant Ethic and the Spirit of Capitalism. English translation by T. Parsons. NY, NY: Scribner. 